\title{
APODIZING OF INTENSE LASER BEAMS WITH SATURABLE DYES
}

\author{
A. PENZKOFER and W. FRÖHLICH \\ Fachbereich Physik der Universität Regensburg, D 8400 Regensburg, Fed. Rep. Germany
}

Received 8 November 1978

\begin{abstract}
The diffraction of laser light by optical components is reduced with graded apertures. The nonlinear transmission of light through saturable absorbers is applied to form soft apertures. Dye cells in front of amplifiers and at the focal region of spatial filters were used to generate smooth beam profiles.
\end{abstract}

Optical components in the light path act as apertures which truncate the light beam and cause diffraction. In the near field behind an aperture $\left(l \lesssim r_{\mathrm{a}}^{2} / \lambda ; r_{\mathrm{a}}\right.$ radius of aperture, $\lambda$ laser wavelength; distance $l \lesssim 25 \mathrm{~m}$ for $r_{\mathrm{a}}=0.5 \mathrm{~cm}$ and $\lambda=1 \mu \mathrm{m}$ ) the Fresnel diffraction modulates the spatial intensity distribution. In the far field $\left(l>r_{\mathrm{a}}^{2} / \lambda\right)$ the Fraunhofer diffraction generates Airy rings.

The distortion of the beam profile by diffraction causes severe problems in laser physics. The profile changes with incident beam diameter and beam shape, with aperture width and distance behind the aperture $[1,2]$. A quantitative analysis of nonlinear optical and spectroscopic effects is difficult with rippled beams. The breakup of light beams in optical media by small scale self-focusing is determined by the width and the depth of the ripples [3-8]. The light intensity in laser amplifiers has to be kept below the self-focusing threshold to avoid damaging of the material and to confine the laser energy within a small divergence angle.

The diffraction effects may be reduced by two different approaches: First, their influence is lowered by increasing aperture diameters. The size of optical components is limited by technical and economical considerations. Second, the diffraction is reduced by properly shaping the spatial intensity distribution of the laser light. Beams with constant intensity across the aperture are strongly diffracted while beams that do not touch the aperture edges suffer no diffraction at the particular opening. At a fixed peak intensity the energy transfer through an aperture is largest for a constant intensity distribution. An optimum beam shape is found when the distortion due to diffraction is tolerably small while the energy flow through the aperture is maximal.

Optical devices with high on-axis transmission and with radially increasing attenuation act as apodized apertures [9]. The diffraction caused by these apodizers is generally small. The beam profile is reshaped so that diffraction in subsequent hard apertures (constant transmission across the opening) is negligibly small.

Several techniques of apodizing laser beams have been reported [10]. Dye cells with radially growing thickness [11], nonuniform metallic films [12,13], photographic plates [13-15], and multilayer dielectric coatings $[13,15]$ were employed. The transmission of light through fringing field Pockels cells and Faraday rotators between polarizers results in the desired soft aperture effect [16]. Annular negative lenses were used to form smooth beam profiles $[17,18]$. Beam dividers using frustrated total internal reflection served the same purpose [19].

In this letter we describe a new technique of apodizing laser beams. We apply the intensity dependent transmission of light through a saturable absorber in a cell of . constant thickness. The central part of the light beam bleaches the dye while the wings are strongly absorbed. The saturable dye is placed in front of hard apertures (e.g. amplifiers) to avoid subsequent diffraction. Laser beams with severe diffraction pattern were smoothed by focusing the light and putting an absorber cell in the focal plane (spatial filtering [20,21] with saturable dye instead of pinhole; the Airy rings of Fraunhofer diffraction are formed in the focal plane). In our experiments 


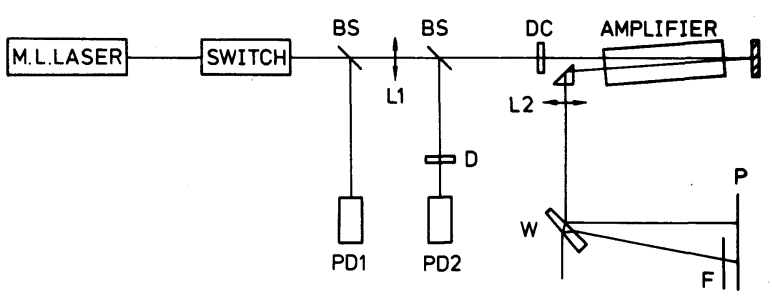

Fig. 1. Experimental set-up. BS, beam aplitters; L1, L2, lenses; DC, apodizing absorber sample; $D$, dye cell for intensity detection; PD1, PD2, photodetectors; W, optical wedge; F, filter; $P$, photographic plate.

with picosecond light pulses the saturable absorber additionally eliminates the background energy content [22] and shortens the pulse duration [23].

The experimental set-up is depicted in fig. 1. A modelocked Nd-glass laser is used to generate a train of picosecond light pulses at $\lambda=1.055 \mu \mathrm{m}$ [24]. The laser is operated at the fundamental transverse mode. An electrooptical shutter selects a single pulse from the pulse train. The separated pulse has a duration of $\Delta t_{\mathrm{L}} \approx 6 \mathrm{ps}$ (fwhm) and an energy of approximately $50 \mu \mathrm{J}$. The intensity of the light pulse is increased with lens L1 $(f$ $=1 \mathrm{~m}$ ). The dye sample DC (Eastman Kodak dye No. 9860) is placed in a region of $I_{0 \mathrm{~L}} \approx 10^{10} \mathrm{~W} / \mathrm{cm}^{2}$ (behind the focal plane). A thin sample of $2 \mathrm{~mm}$ thickness was used to avoid wave front distortion by the nonlinear refractive index. The intensity of the light pulse was monitored by measuring the energy transmission through a separate absorber cell D [25]. The small signal transmission of the dye in the apodizing sample DC was chosen to be $T_{0}=10^{-4}$. The central part of the laser beam passes the sample without remarkable attenuation while the wings are strongly absorbed. The filtered pulse traverses twice an Nd-glass amplifier (length $23 \mathrm{~cm}$, diameter $1.27 \mathrm{~cm}$, amplification in the double pass $\sim 100$ ). The light beam behind the amplifier is collimated with lens $\mathrm{L} 2(f=2 \mathrm{~m})$. The spatial intensity distribution is analysed with photographic plates (Kodak Spectroscopic plates type 1Z). To calibrate the plates the laser beam is split into two parts with a wedge and one part is attenuated (factor of 3 ) with a filter. The beam profiles at various distances behind the amplifier were analysed by studying the microdensitometer traces. The measured beam profiles were smooth when the dye sample DC was in its position. Without the dye the output profile was found to be rippled.

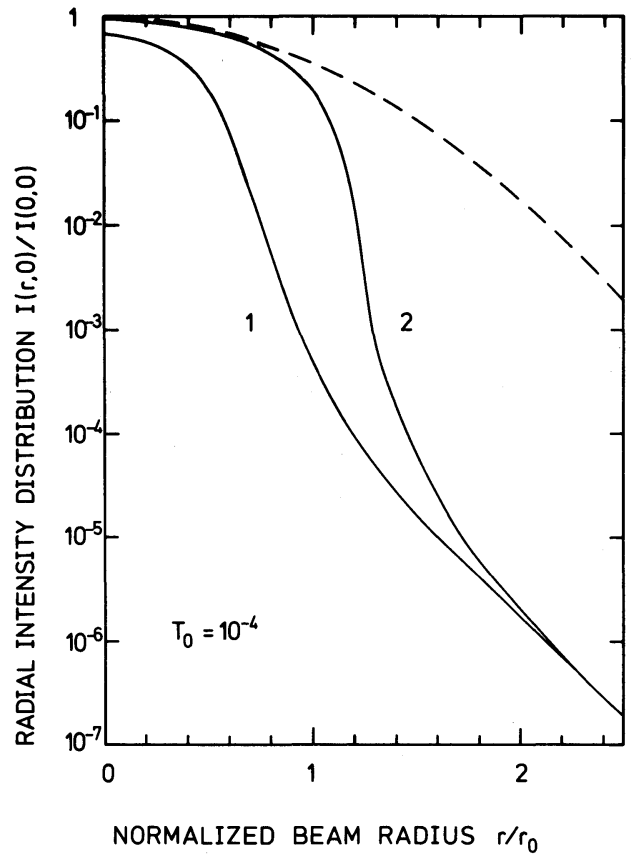

Fig. 2. Reshaping of laser beams in a saturable absorber. Dashed curve, incident gaussian pulse. Solid curves, transmitted radial intensity distribution at $t^{\prime}=0$ for input peak intensities of $I_{0 \mathrm{~L}}=2 \times 10^{9} \mathrm{~W} / \mathrm{cm}^{2}$ (curve 1) and $10^{10} \mathrm{~W} / \mathrm{cm}^{2}$ (curve 2). Small signal transmission of the sample $T_{0}=10^{-4}$.

The reshaping of gaussian input beams in the dye sample is shown in fig. 2. The input pulse shape $\left(I\left(r, t^{\prime}\right)\right.$ $=I_{0} \exp \left[-\left(t^{\prime} / t_{0}\right)^{2}-\left(r / r_{0}\right)^{2}\right]$ at $t^{\prime}=t-c z / n=0$ is given by the dashed curve. The transmitted pulse shapes at $t^{\prime}=0$ correspond to $I_{0 \mathrm{~L}}=10^{10} \mathrm{~W} / \mathrm{cm}^{2}$ and $2 \times 10^{9} \mathrm{~W} / \mathrm{cm}^{2}$, respectively. An initial dye transmission of $T_{0}=10^{-4}$ is used. At $I_{0 \mathrm{~L}}=10^{10} \mathrm{~W} / \mathrm{cm}^{2}$ (curve 2) the central part of the beam is unaffected up to a radius of $r \approx 0.9 r_{0}$ and then drops down rapidly. For $r \geqslant 2.5 r_{0}$ the input pulse is reduced by the constant factor of $T_{0}=10^{-4}$. At $I_{0 \mathrm{~L}}=2 \times 10^{9} \mathrm{~W} / \mathrm{cm}^{2}$ (curve 1) the central part of the beam is slightly attenuated and the shape of the beam changes more gradually. The pulse duration is shortened nearly by a factor of two at this intensity value (see fig. 3 ). The curves in figs. 2 and 3 are calculated according to ref. [23].

The modulation of the beam profiles by hard apertures depends on the intensity value $I\left(r_{\mathrm{a}}\right)$ at the aperture edge. The modulation was found to be tolerably small (no small scale self-focusing problems) for $I\left(r_{\mathrm{a}}\right) / I(0) \leqslant 10^{-4}[1,2,26]$. The energy transfer through 


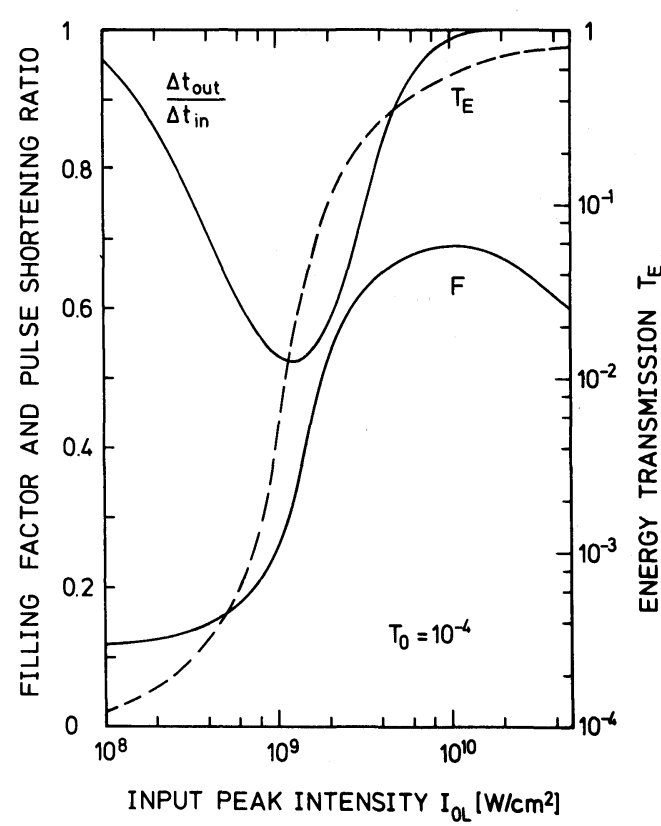

Fig. 3. Characteristics of apodizing absorber. Curve $\mathrm{F}$ represents the filling factor of subsequent hard apertures. Curve $T_{\mathrm{E}}$ gives the energy transmission through the absorber. The pulse shortening by the dye is shown by the curve $\Delta t_{\text {out }} / \Delta t_{\text {in }}$.

a hard aperture depends on the pulse shape. We define a filling factor $F$ for an intensity distribution $I(r)$ as the ratio $F=\int_{0}^{r} \mathrm{~F} 2 r I(r) \mathrm{d} r /\left[r_{\mathrm{F}}^{2} I(0)\right]$ of the actual energy transmission to the energy transmission of a rectangular pulse. $r_{\mathrm{F}}$ is the aperture radius at which $I(r) / I(0)=$ $=10^{-4}$. The curve labeled $\mathrm{F}$ in fig. 3 shows the filling ratio of light beams apodized in the dye sample versus the input peak intensity $\left(T_{0}=10^{-4}\right)$. An optimum value of $F \approx 0.7$ is found at $I_{0 \mathrm{~L}} \approx 10^{10} \mathrm{~W} / \mathrm{cm}^{2}$. For a gaussian pulse $F$ is approximately 0.1 .

The energy transmission through the sample DC is presented by curve $T_{\mathrm{E}}$ in fig. 3. $T_{\mathrm{E}}$ increases with $I_{0 \mathrm{~L}}$ and reaches a value of 0.5 at $10^{10} \mathrm{~W} / \mathrm{cm}^{2}$. The pulse shortening ratio $\Delta t_{\text {out }} / \Delta t_{\text {in }}$ for gaussian input pulses is also depicted in fig. 3. For $I_{0 \mathrm{~L}} \geqslant 10^{10} \mathrm{~W} / \mathrm{cm}^{2}$ the pulse durations (fwhm) are not altered. At $I_{0 \mathrm{~L}} \approx 2$ $\times 10^{9} \mathrm{~W} / \mathrm{cm}^{2}$ the pulses are remarkably shortened and the filling factor is high: The effects of pulse shortening and apodizing occur simultaneously.

In fig. 4 two beam profiles are presented which were measured $4 \mathrm{~m}$ behind the pumped amplifier. Curve a shows a microdensitometer trace when the absorbing dye was removed. Curve $\mathrm{b}$ was obtained with

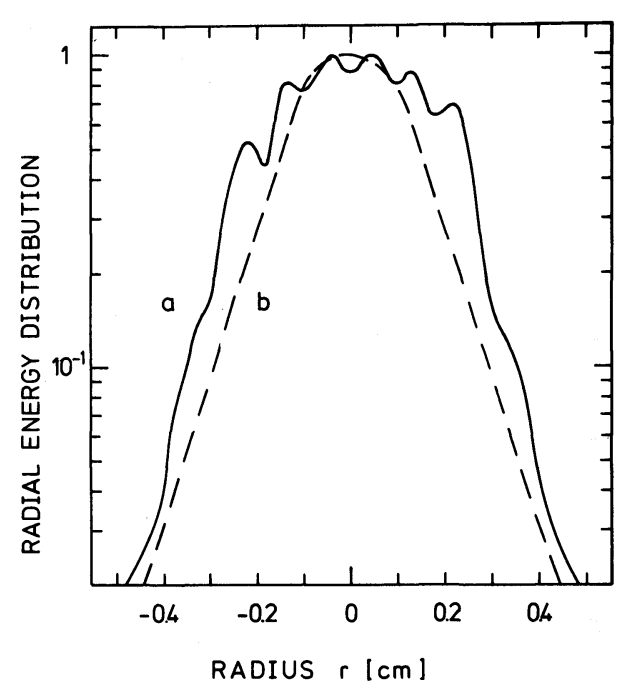

Fig. 4. Radial energy distribution of laser light $4 \mathrm{~m}$ behind amplifier (diameter $1.27 \mathrm{~cm}$ ). Curve a, without apodizing dye; curve $b$, with bleachable dye. $I_{0 \mathrm{~L}} \approx 10^{10} \mathrm{~W} / \mathrm{cm}^{2}, T_{0}=10^{-4}$.

the dye sample in its position $\left(I_{0 \mathrm{~L}} \approx 10^{10} \mathrm{~W} / \mathrm{cm}^{2}\right.$ at $\mathrm{DC})$. The reduction of the diffraction effects by the saturable absorber is clearly seen.

The far field diffraction pattern is determined by the Fourier transform of the electric field strength at the aperture. In fig. 5 three Fraunhofer diffraction shapes are presented. Curve 1 (dotted) corresponds to an undisturbed gaussian pulse (it remains gaussian). Curve 2 belongs to the apodized beam at $I_{0 \mathrm{~L}}=10^{10}$ $\mathrm{W} / \mathrm{cm}^{2}$ (Curve 2 in fig. 2) which is truncated at $r_{\mathrm{a}}$ $=r_{\mathrm{F}}\left[I\left(r_{\mathrm{F}}\right) / I(0)=10^{-4}\right]$. The fringe height is mainly due to the rapid decrease of intensity between $r \approx r_{0}$ and $r=r_{\mathrm{F}}$ (caused by the saturable dye). Curve 3 shows the diffraction pattern of an unapodized gaussian pulse (dashed curve in fig. 2) which is truncated at the same aperture radius as curve $2\left[I\left(r_{\mathrm{a}}\right) / I(0) \approx 0.12\right]$. The far-field diffraction rings of curves 2 and 3 are not remarkably different. The intensity of the rings may be lowered by putting an additional saturable absorber cell in the path of the beam at the far field. Under our experimental conditions far field diffraction occurs for distances $l \gtrsim 40 \mathrm{~m}$ and we are more concerned with near field diffraction.

The near field diffraction was studied by solving the Fresnel-Kirchhoff formula [27] 


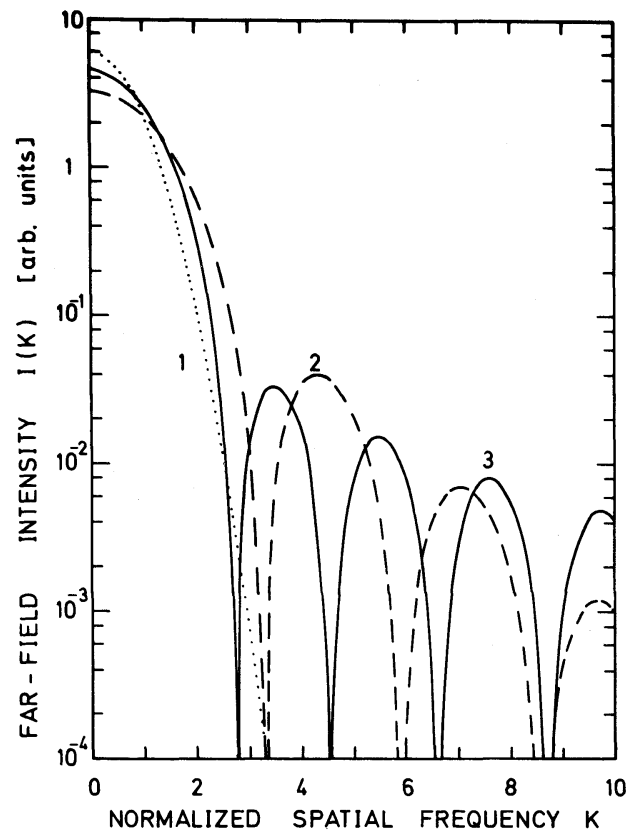

Fig. 5. Far-field intensity distribution. Curve 1 (dotted), no aperture in the path of a gaussian beam. Curve 2 (dashed), diffraction pattern of apodized and truncated light pulse $\left(I_{0 \mathrm{~L}}=10^{10} \mathrm{~W} / \mathrm{cm}^{2}, T_{0}=10^{-4}, I\left(r_{\mathrm{a}}\right) / I(0)=10^{-4}\right)$. Curve 3 (solid), diffraction fringes of truncated gaussian pulse $\left(I\left(r_{\mathrm{a}}\right) / I(0)\right.$ $=0.12$ ). The normalized spatial frequency is $K=k R r_{0} / l$, where $R$ radius at distance $l$.

$$
\begin{aligned}
& E\left(t^{\prime}, R, l\right) \\
& =-\frac{\mathrm{i} 2}{\lambda} \int_{0}^{r} \int_{0}^{\pi} \frac{\exp \left[\mathrm{i} k\left(r^{2}+R^{2}+l^{2}-2 R r \cos \varphi\right)^{1 / 2}\right]}{r^{2}+R^{2}+l^{2}-2 R r \cos \varphi} \\
& \quad \times r l E\left(t^{\prime}, r, 0\right) \mathrm{d} \varphi \mathrm{d} r
\end{aligned}
$$

by numerical methods. Plane wavefronts at a circular aperture of radius $r_{\mathrm{a}}$ are assumed. $l$ is the distance behind the aperture, $R$ and $r$ are the radial coordinates at $l$ and at the aperture, respectively; $k=2 \pi / \lambda$ is the wave vector of the laser light. $E$ represents the electrical field strength. In fig. 6 the dependence of the onaxis intensity $I\left(t^{\prime}=0, R=0, l\right)$ on the propagation distance $l$ is depicted. The upper part (a) presents a gaussian pulse which is truncated at $I\left(r_{\mathrm{a}}\right) / I(0)=10^{-4}$. A slight modulation is seen out to a distance of $l \approx 10 \mathrm{~m}$. The dashed curve in fig. $6 \mathrm{~b}$ shows the apodized pulse which is truncated at $I\left(r_{\mathrm{a}}\right) / I(0)=10^{-4}$ (pulse has the

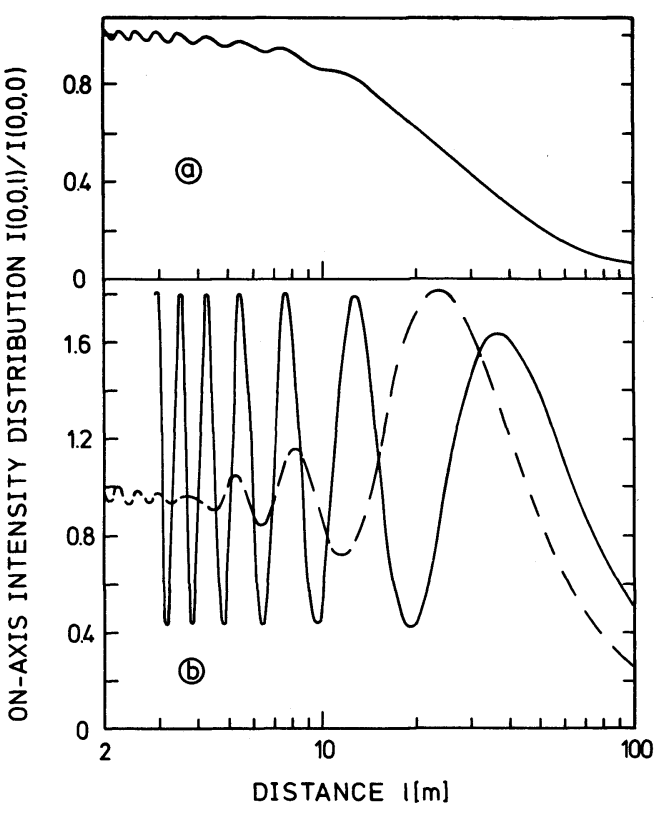

Fig. 6. Near-field on-axis intensity distribution versus distance from aperture. (a) Gaussian pulse truncated at $I\left(r_{\mathrm{a}}\right) / I(0)=10^{-4}$, (b) dashed curve, apodized truncated pulse $\left(I_{0 \mathrm{~L}}=10^{10} \mathrm{~W} / \mathrm{cm}^{2}\right.$, $\left.T_{0}=10^{-4}, I\left(r_{\mathrm{a}}\right) / I(0)=10^{-4}\right)$; solid curve, gaussian pulse truncated at $I\left(r_{\mathrm{a}}\right) / I(0)=0.12 . r_{\mathrm{a}}=0.635 \mathrm{~cm}, \lambda=1.055 \mu \mathrm{m}$.

same parameters as curve 2 of fig. 5). Up to a distance of $l \approx 5 \mathrm{~m}$ the modulation depth of the apodized beam is only slightly larger than for the gaussian pulse of fig. 6a. It should be noted that the filling ratio of the apodized pulse is nearly a factor of 7 larger than the filling ratio of the gaussian pulse. The solid curve in fig. $6 \mathrm{~b}$ depicts the behaviour of a gaussian pulse which is cut-off at $I\left(r_{\mathrm{a}}\right) / I(0)=0.12$ (see curve 3 of fig. 5). The modulation depth is enormous. For large distances the apodized beam (dashed curve) approaches the behaviour of the truncated gaussian beam (solid curve). The steep slope of the reshaped pulse is responsible for this fact as was pointed out for the Fraunhofer diffraction of fig. 5. The modulation frequency decreases with distance $l$ and ceases in the far field $(l>40 \mathrm{~m})$. In the calculations $r_{\mathrm{a}}=0.635 \mathrm{~cm}$ was used according to the radius of our amplifier rod.

The radial intensity distribution of the two light pulses discussed in fig. $6 \mathrm{~b}$ is presented in fig. 7 for the distances $l=2.5 \mathrm{~m}$ and $l=5 \mathrm{~m}$. The apodized pulse (dashed curves) shows a rather smooth modulation. The gaussian pulse truncated at $I\left(r_{\mathrm{a}}\right) / I(0)=0.12$ (solid 


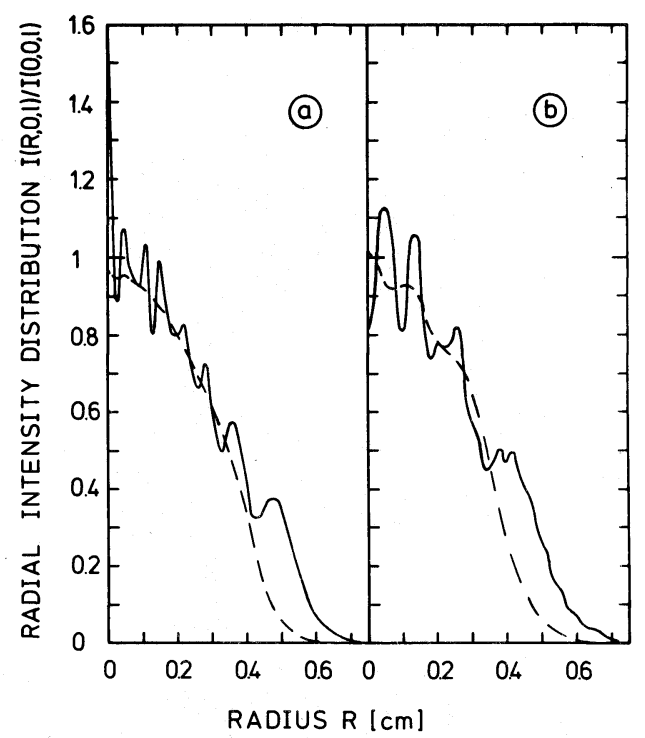

Fig. 7. Radial intensity distributions in the near field. Dashed curves, apodized pulse; solid curves, truncated gaussian; as in fig. $6 \mathrm{~b}$. Aperture radius $r_{\mathrm{a}}=0.635 \mathrm{~cm}$, wavelength $\lambda=1.055 \mu \mathrm{m}$; distance behind aperture $l=2.5 \mathrm{~m} \mathrm{(a)}$ and $5 \mathrm{~m} \mathrm{(b)}$.

curves) is severely rippled.

Light beams with strong Fresnel diffraction pattern may be smoothed by using spatial filters $[20,21]$. We tested the possibility of spatial filtering with a saturable dye in the focal plane of the filter instead of a pinhole. Rather long focal lengths $f$ had to be used to set the proper peak intensity $I_{0 \mathrm{~L}}$ in the focal plane $\left(5 \times 10^{9} \mathrm{~W} / \mathrm{cm}^{2} \leqslant I_{0 \mathrm{~L}} \leqslant 5 \times 10^{10} \mathrm{~W} / \mathrm{cm}^{2}\right.$, for $T_{0}$ $=10^{-4}$ ). We used $f=6 \mathrm{~m}$ after the pumped amplifier. The beam profile behind the spatial filter was found to be very smooth. The application of a dye cell in the focal plane of the laser pulse may be helpful to clean the spatial distribution before entering the sample under investigation.

In conclusion we wish to state that we have introduced a new technique of apodizing intense light beams. The technique uses bleachable dyes to reduce diffraction effects in subsequent optical components. Spatial filters with saturable absorbers instead of pinholes were applied to clean severely fringed pulses.

The authors are indepted to Professor M. Maier for valuable discussions.

\section{References}

[1] A.J. Campillo, J.E. Pearson, S.L. Shapiro and N.J. Terrell, Jr., Appl. Phys. Letters 23 (1973) 85.

[2] G.R. Hadley, IEEE J. Quant. Electr. QE-10 (1974) 603.

[3] V.I. Bespalov and V.I. Talanov, Sov. Phys. JETP-Letters 3 (1966) 307.

[4] A.J. Campillo, S.L. Shapiro and B.R. Sydam, Appl. Phys. Letters 23 (1973) 628.

[5] J.A. Fleck, Jr. and C. Layne, Appl. Phys. Letters 22 (1973) 467.

[6] J.F. Holzrichter and D.R. Speck, J. Appl. Phys. 47 (1976) 2459.

[7] E.S. Bliss, J.T. Hunt, P.A. Renard, G.E. Sommargren and H.J. Weaver, IEEE J. Quant. Electr. QE-12 (1976) 402.

[8] J.C. Guyot, A. Bettinger and D. Auric, Rev. de Phys. Appl. 13 (1978) 198.

[9] P. Jacquinot and B. Roizen-Dossier, Progress in Optics, Vol. 3, ed. E. Wolf (Wiley, New York, 1964) p. 29.

[10] V.R. Costich and B.C. Johnson, Laser Focus, Sep. 1974, p. 43.

[11] Lawrence Livermore Laboratory Laser Fusion Program Semi-annual Report, July-Dez. 1972, UCRL-50021-72-2.

[12] Lawrence Livermore Laboratory Laser Fusion Program Semi-annual Report, July-Dez. 1973, UCRL-50021-73-2.

[13] K.A. Brueckner, B. Guscott, S. Jorna, K. Moncur and L. Siebert, NBS-Special Publication No. 414, p. 2 (US. Gov. Print Office, Washington 1974).

[14] A.J. Campillo, B. Carpenter, B.E. Newman and S.L. S.L. Shapiro, Opt. Comm. 10 (1974) 313.

[15] J. Sources, S. Kumpan and J. Hoose, Appl. Opt. 13 (1974) 2081.

[16] W.W. Simmons, G.W. Leppelmeier and B.C. Johnson, Appl. Opt. 13 (1974) 1629.

[17] B.J. Feldman and S.J. Gitomer, Appl. Opt. 15 (1976) 1379.

[18] L. Seppala, Appl. Opt. 16 (1977) 1482.

[19] J.-C. Diels, Appl. Opt. 14 (1975) 2810.

[20] A. Bettinger, C. Charles, J. Osmalin and J.G. Giraud, Opt. Comm. 18 (1976) 176.

[21] W.W. Simmons, S. Guch, Jr., F. Rainer and J.E. Murray, IEEE J. Quant. Electr. QE-11 (1975) 30D.

[22] J. Wiedmann and A. Penzkofer, Opt. Comm. 25 (1978) 226.

[23] A. Penzkofer, Opto-Electronics 6 (1974) 87.

[24] A. Laubereau and W. Kaiser, Opto-Electronics 6 (1974) 1.

[25] A. Penzkofer, D. von der Linde and A. Laubereau, Opt. Comm. 4 (1972) 377.

[26] Lawrence Livermore Laboratory Laser Fusion Program Semi-annual Report, Jan.-June 1973, UCRL-50021-73-1.

[27] A. Sommerfeld, Optics, Lectures on theoretical Physics, Vol. 4 (Academic Press, New York, 1967). 